\title{
Carriage and Antibiogram of Methicillin Resistant Staphylococcus Aureus among Admitted Patients of a Surgical Ward in A Tertiary Hospital, South Western Uganda
}

Musinguzi Benson ( $\square$ musinguzibenson6@gmail.com )

Kampala International University https://orcid.org/0000-0002-1211-4617

Pross Mugimba

Mbarara University of Science and Technology

Andrew Baguma

Kabale University

Herbert Itabangi

Busitema University

Joel Bazira

Mbarara University of Science and Technology

\section{Research Article}

Keywords: MRSA carriage, Susceptibility patterns and Host factors.

Posted Date: June 2nd, 2021

DOI: https://doi.org/10.21203/rs.3.rs-529905/v1

License: (a) (i) This work is licensed under a Creative Commons Attribution 4.0 International License.

Read Full License 


\section{Abstract}

Background: Methicillin Resistant Staphylococcus aureus (MRSA) is one of the most common nosocomial infections affecting post-surgery patients. Antimicrobial resistance prolongs the duration of hospitalization. We sought to determine the MRSA carriage among patients admitted to surgical ward in a tertiary hospital in South Western Uganda.

Methods: Total of 46 participants were included in the study, swabbed at four different sites: nares, hands, perineum and surgical wound site. Identification of Staphylococcus aureus was done by standard conventional microbiological methods. Antibiotic susceptibility testing was done using disk diffusion method, except for Vancomycin were MIC was used as per CLSI guidelines. Using these results, prevalence of MRSA was determined. The on admission were followed up for 7days to determine the incidence of MRSA. Site carriage rate of MRSA was determined using drug susceptibility test (DST) test results. Questionnaire was used to collect data on factors associated with MRSA.

Results: The prevalence of MRSA carriage at admission was 18/46 (39.1\%). Among patients who were MRSA negative at admission, the incidence of MRSA carriage during hospitalization was $13 / 26$ (50\%). Of the $122 \mathrm{~S}$. aureus isolates, resistance to Oxacillin was 55(45.1\%), Ciprofloxacin 50(41.0\%), Ceftriaxone 46(37.7\%), Methicillin 44(36.1\%), Levofloxacin 39(32.0), Imipenem 13(10.7\%) and Vancomycin 3(2.5\%).

The site with the highest carriage rate was the Nares 16/77 (20.8\%), Hands 14/77(18.2\%), Perineum $15 / 77(19.5 \%)$, surgical wound site $9 / 77(11.7 \%) .80 \%$ of the isolates were MDR. The factors measured were not statistically associated to MRSA carriage outcome.

Conclusion: There was high MRSA carriage among patients on surgical ward. Resistance to commonly used antibiotics was high, $80 \%$ of the isolates were MDR. Therefore, there is the need for continuous surveillance to monitor aetiology and antimicrobial susceptibility patterns in order to guide the empirical use of antimicrobials. Sex was the only factor associated with MRSA carriage. Further studies should be done to fully assess factors associated to MRSA.

\section{Introduction}

S. aureus a gram positive bacterium is a commensal of human skin and mucosal areas. S. aureus colonizes different sites of the body, the primary areas colonized are, anterior nares of the nose, skin, perineum, gastrointestinal tract, vagina and axilla [1]. S. aureus causes skin, bone, soft tissue infections, urinary tract infection, pneumonia and bacteremia in community and hospital setting [2]. Despite the availability of effective antimicrobial agents to treat $S$. aureus infections, it continues to be a major cause of morbidity and mortality worldwide. S. aureus has proven to be among the most persistent of pathogens in the healthcare and community setting $[3,4]$.

Methicillin a modified penicillin, designed to resist the destructive action of the staphylococcal $\beta$ lactamase, became available for therapeutic use in 1959, but in 1961, S. aureus isolates had acquired 
resistance to methicillin and the first methicillin-resistant $S$. aureus (MRSA) case was reported [1]. Methicillin-resistant $S$. aureus (MRSA) is a $S$. aureus that has become resistant to beta-lactam antibiotics [5]. Methicillin resistance is due to the mecAgene, on the staphylococcal cassette chromosome mec(SCCmec) that codes for a78-kDa penicillin binding protein (PBP2a), with decreased affinity to methicillin and all beta-lactam antibiotics[6]. There is additional morbidity and mortality for MRSA patients as compared to patients infected with methicillin-susceptible S. aureus (MSSA) [7].

Two major forms of MRSA have been identified, the community acquired (CA-MRSA) which infect patients with no established risk factor and the hospital acquired (HA-MRSA) which infect patients with risk factors like recent hospitalization, having under gone a surgical procedure, immune suppression or underlying chronic disease, recent use of broad-spectrum antimicrobials, use of invasive medical devices (urinary catheter, nasogastric tube, or IV catheter), and chronic diseases leading to wounds or skin breakdown $[8,9]$. MRSA carriage is asymptomatic, but can be a source of infection to its host and may play role in an outbreak in a clinical setting [10]. The incidence of hospital acquired MRSA continues to rise globally. In a study by Abdulgader et al, he noted that, it was estimated that MRSA infections within the health care setting alone affected more than 150,000 patients annually in the European Union, and in the United States of America, 80,461 invasive MRSA infections and 11,285 related deaths occurred in 2011[6]. In a study done in multiple sites in Kenya, the prevalence of multidrug resistant MRSA was $60 \%$ with relatively high resistance to erythromycin, gentamicin and oxacillin [11]. In Uganda $10 \%$ of the surgical procedures become septic with $S$. aureus being the most frequent pathogen isolated. MRSA can become MDR posing a threat of outbreaks which may be difficult to control once introduced into the hospital setting. Current therapeutic options for MRSA are limited to few potentially toxic and expensive drugs like teicoplanin, vancomycin, linezolid, daptomycin and streptogramins. [5]. There is therefore the need for early and accurate diagnosis of MRSA in order to prevent and control outbreaks. The prevalence of MRSA among clinical isolates in previous study done in the same setting (MRRH) was high (38\%) in 2014[8], however there was a significant drop in the prevalence to $2.8 \%$ in 2016[12], therefore need for continuous surveillance to monitor $S$. aureus infections as well as determine the role of $S$. aureus carriage in infection so that control measures can be put in place.

\section{Materials And Methods}

\section{Type of study.}

This was a prospective quantitative study in which, prevalence at admission and incidence of MRSA carriage during hospitalization, susceptibility patterns of MRSA and host factors associated with MRSA were determined among patients admitted of a surgical ward in Mbarara Regional Referral Hospital (MRRH) between June and August 2019.

\section{Study population.}

The study targeted patients that had undergone surgery and were due for admission on surgical ward. A total of 46 participants were included in the study. 


\section{Inclusion/exclusion criteria.}

All patients who had undergone surgery and were due for admission on surgical ward, on consenting to participate, were included in the study. Patients who had been admitted for more than $24 \mathrm{hrs}$, transfers in from different wards/hospitals and patients discharged before third day, were not included in the study.

\section{Sample collection and laboratory processing.}

Specialists, residents and interns were incorporated into the study to facilitate collection of specimens. Using a sterile swab for each site, the four different sites were swabbed and each swab put in a container containing transport medium, labelled with the participant number and the initial of the site that was swabbed. These samples were transported to the laboratory for analysis. Thereafter, four subsequent samples from the same areas were taken off after $48 \mathrm{hrs}$ during the time of admission for a maximum of 7 days. Blood agar and mannitol salt agar were used for the isolation of $S$. aureus from the samples collected. Gram stain, catalase, coagulase and DNase test were performed on colonies suggestive of $S$. aureus to confirm that the organism in question was indeed $S$. aureus.

\section{Antimicrobial susceptibility test.}

The Antimicrobial susceptibility test was performed on colonies identified as $S$. aureus. DST was performed using the disc diffusion method on Mueller Hinton Agar (MHA) plates as recommended by the Clinical and Laboratory Standards Institute (CLSI). The antibiotic disks used were, methicillin, oxacillin, ciprofloxacin, Ceftriaxone, imipenem, levofloxacin. The minimum inhibitory concentration of vancomycin were determined by agar dilution method in MHA. Different dilutions of vancomycin used were $0.0625 \mu \mathrm{g} / \mathrm{ml}$ to $64 \mu \mathrm{g} / \mathrm{ml}$. results were interpreted according to the protocol of CLSI 2018 guidelines were $\leq 4 \mu \mathrm{g} / \mathrm{ml}$ was sensitive, $4-6 \mu \mathrm{g} / \mathrm{ml}$ intermediate and $\geq 16 \mu \mathrm{g} / \mathrm{ml}$ was resistant. Quality control was achieved by following the standard operating procedures (S.O.Ps), S. aureus ATCC 29213 as an oxacillin susceptible strain and S. aureus ATCC 43300 as an oxacillin resistant strain were included during laboratory tests in the study.

\section{Statistical analysis.}

Descriptive statistics of continuous demographic variables were summarized and presented as mean with respective standard deviation, frequencies and percentages for categorical descriptive variables, bivariate analysis was done and $p$-value $<0.05$ was taken as significant.

\section{Results}

\section{Demographic characteristics of study participants.}

As shown in table 1, forty-six (46) participants were included in the study. The majority of study participants were male $(30,65.2 \%)$. Most of the study participants were 18 years and above $(19,41.3 \%)$, children were $(15,32.6 \%)$ while infants were $(12,26.1 \%)$ as shown in the table 1 below. 
Table 1: Demographic characteristics of study participants $(N=46)$.

\begin{tabular}{|ll|}
\hline Variable & $\begin{array}{l}\text { Summary of measures } \\
\text { n_(\%). }\end{array}$ \\
Female & $16(34.8)$ \\
Male & $30(65.2)$ \\
Age Category & \\
Infants & $12(26.1)$ \\
Children & $15(32.6)$ \\
Adults & $19(41.3)$ \\
\hline
\end{tabular}

\section{Prevalence of MRSA carriage on admission.}

Out of 46 participants, $39.1 \%$ (18) had MRSA while $60.9 \%$ (28) had no MRSA prior to admission as shown in figure 1 below.

\section{Incidence of MRSA carriage during hospitalization.}

Out of 28 participants who had MRSA prior to admission; only 26 participants were followed up for 7days and 50\% (13) participants developed MRSA while the remaining 50\% (13) did not develop MRSA as shown in the figure 2 below:

\section{Antibiotic Susceptibility patterns of the isolated $S$. aureus.}

The total number of samples collected were 308 swabs from the different sites that is nares, perineum, hands and surgical wound site. The number of $S$. aureus isolates from the collected samples were 122 isolates. All the $S$. aureus isolates were subjected to Antimicrobial susceptibility testing by disc diffusion method and MIC for vancomycin. The antibiotics tested were Methicillin $(5 \mu \mathrm{g})$, Oxacillin $(1 \mu \mathrm{g})$, Ceftriaxone $(30 \mu \mathrm{g})$, Ciprofloxacin $(5 \mu \mathrm{g})$, Levofloxacin $(5 \mu \mathrm{g})$, Vancomycin $(30 \mu \mathrm{g})$, Imipenem $(10 \mu \mathrm{g})$. Figure 3 below shows the DST pattern of the S. aureus isolates to the different antibiotics.

\section{Site with highest rate of MRSA carriage.}

Samples were collected from four different sites that is the nares, hands, perineum and the surgical wound site. The total number of samples collected from each site were 77 swabs. These findings were obtained from 31 participants who were MRSA positive. Among the 31 participants, some of them had MRSA carriage at more than one site. One participant had carriage at all the four sites 
$1(3.2 \%), 3(9.7 \%)$ at three sites, majority at two sites $14(45.1 \%)$, while $13(42 \%)$ had carriage at only one site. The site with highest rate of MRSA carriage was the nares as demonstrated in the figure 4 below.

\section{Factors Associated with MRSA carriage}

For all the factors measured, only sex was associated to MRSA. The odds were 0.19 times higher that a male participant would get MRSA compared to a female. This association was statistically significant since the $p$-value $=0.04$ was less than 0.05 as shown in table 2 below.

\section{Table 2: Factors associated with MRSA carriage.}

\begin{tabular}{|c|c|c|c|c|}
\hline \multicolumn{5}{|c|}{ MRSA Result n (\%) } \\
\hline Variable & Positive & Negative & unadjusted $\mathrm{OR}(95 \% \mathrm{CI})$ & $P$ value \\
\hline \multicolumn{5}{|l|}{ Sex } \\
\hline Female & $14(45.2)$ & $2(13.3)$ & 1.00 & \\
\hline Male & $17(54.8)$ & $13(86.7)$ & $0.19(0.03-1.08)$ & 0.04 \\
\hline \multicolumn{5}{|c|}{ Blood Transfusion } \\
\hline Yes & $6(19.35)$ & $1(6.7)$ & 1.00 & \\
\hline No & $25(80.65)$ & $14(93.3)$ & $0.30(0.03-2.89)$ & 0.27 \\
\hline \multicolumn{5}{|c|}{ Anesthesiadone } \\
\hline General & $21(67.74)$ & $11(73.33)$ & 1.00 & \\
\hline Local & $7(22.58)$ & $4(26.67)$ & $0.92(0.22-3.90)$ & 0.91 \\
\hline Spinal & $3(9.68)$ & $0(0.00)$ & & 0.23 \\
\hline \multicolumn{5}{|c|}{ Age category } \\
\hline Infants & $10(32.26)$ & $2(13.33)$ & 1.00 & \\
\hline Children & $8(25.81)$ & $7(46.67)$ & $0.23(0.03-1.62)$ & 0.11 \\
\hline Adults & $13(41.94)$ & $6(40.00)$ & $0.43(0.07-2.77)$ & 0.36 \\
\hline \multicolumn{5}{|l|}{ Treatment } \\
\hline Combined & $19(61.29)$ & $10(66.67)$ & 1.00 & \\
\hline Single & $8(25.81)$ & $4(26.67)$ & $1.05(0.25-4.45)$ & 0.94 \\
\hline Others & $4(12.9)$ & $1(6.67)$ & $2.10(0.20-22.5)$ & 0.52 \\
\hline Positive & $1(3.23)$ & $1(6.67)$ & 1.00 & \\
\hline Negative & $30(96.77)$ & $14(93.33)$ & $2.14(0.12-38.3)$ & 0.6 \\
\hline
\end{tabular}

Treatment combined $=$ Ceftriaxone + metronidazole. Single= Ceftriaxone only.

Others: Ampiclox, gentamycin and flucamox 


\section{Discussion}

Out of the 46 participants that were included in the study, the prevalence of MRSA colonization at admission was $39.1 \%$. Prevalence of MRSA colonization was highest among infants at $58.3 \%$ while among the children and adults, prevalence of MRSA colonization was at $26.7 \%$ and $36.8 \%$ respectively. This study is in agreement with the study carried out in MRRH by Stanley et al 2014 which reported the prevalence of MRSA among clinical isolates causing infections as 31.3\% [8]. These finding also agree with those from a study carried out in Mulago hospital were out of $54(28.7 \%)$ S. aureus isolates, $31.5 \%$ were MRSA as determined by PCR [13]. These findings however don't agree with the findings carried out in a Ugandan regional referral hospital were the prevalence of MRSA was at 2.8\%[12] this could be due to the fact that the study design were different. The high prevalence of MRSA among infants could be attributed to maternal colonization which is a risk factor for $S$. aureus carriage in infants. In a study carried out to determine transmission of Staphylococcus aureus between mothers and infants in an African setting, colonization among infants was at $42 \%$ [14]. The high prevalence of MRSA colonization could also be due to traditional risk factors associated with treatment in healthcare setting [15].

The incidence of MRSA colonization during hospitalization was 13(50\%) out of the 26 participants that were followed up. Incidence of MRSA colonization was highest among infants at 3(75\%), among adults incidence was at 6(54.5\%) and lowest among children at 4(36.4\%). The incidence of HA-MRSA was high this could be attributed to the high prevalence of MRSA colonization which puts patients admitted at risk of colonization due to the risk factors associated to MRSA spread with in the healthcare setting. In a study assessing the efficacy of Active Surveillance culturing (ASC) in an environment endemic for MRSA, 22,000 surgical patients were divided into intervention and control groups. Despite identification of 300 patients who were asymptomatic carriers of MRSA, the incidence of MRSA was $57 \%$ during hospitalization therefore the need of a weekly surveillance system [16].

The S. aureus isolates from the collected samples were 122 isolates. In this study, $80 \%$ of MRSA isolates were MDR. In a similar study carried out in Mulago hospital, $70.6 \%$ of the isolates were resistant to Ciprofloxacin, this was high compared to the $41.0 \%$ obtained in this study. All the isolates in that particular study were susceptible to Vancomycin which is in agreement with study carried out in MRRH 2016[12,13], the findings of this study don't agree. Three (2.5\%) of S. aureus were Vancomycin resistant while Vancomycin intermediate S. aureus were 14(11.5\%). A study by Kahsay et al also indicated resistance to Vancomycin at 5.6\% [17]. Strikingly, Vancomycin is one of the drugs used to treat MRSA [18], there is therefore need for continuous surveillance to monitor aetiology and antimicrobial susceptibility pattern to guide empirical use of antibiotics.

Of all the 77 samples collected from each site, the commonest site colonized by MRSA was the nares at $16(20.8 \%)$, followed by perineum at $15(19.5 \%)$, hand was at $14(18.2 \%)$ and finally the surgical wound site was the least colonized at 9 (11.7\%). These findings were obtained from 31 participants who were MRSA 
positive. Among the 31 participants, some of them were colonized at more than one site. One participant was colonized at all the four sites $1(3.2 \%), 3(9.7 \%)$ at three sites, majority at two sites $14(45.1 \%)$, while $13(42 \%)$ were colonized at only one site. These findings were in agreement with other studies carried out in different setting. In another study, results were as follows: at the time when MRSA was first detected among study participants, the most frequent sites positive for MRSA were a skin lesion (110 patients, $41 \%$ ), the anterior nares (109 patients, $41 \%$ ), and the throat (102 patients, $38 \%$ ). In $26 \%, 17 \%$, and $17 \%$ of the patients in another study, a skin lesion, the anterior nares, and the throat, respectively, were the only sites where MRSA was seen $[19,20]$. Colonization at different sites can be attributed to the fact that MRSA can be transmitted through hands, clothes or equipment of healthcare workers [21]. All MRSA carriers are not the same; carriage may be transient, intermittent, or persistent for months to years. Persistent carriers are more heavily colonized frequently at multiple sites and are more likely to transmit the infection to others and more likely to become infected themselves than transient carriers [22].

Previous studies done have shown that risk factors associated with developing a methicillin resistant bacteremia include: age, prolonged hospitalization, prior antimicrobial treatment, urinary catheterization, nasogastric tube placement, HIV and previous surgery $[16,23]$. In a study carried out by Soltani at el, factors associated with MRSA nasal carriage included gender ( $P$ value 0.001 ), age of less than four years ( $P$ value 0.016$)$, number of individuals in the family ( $P$ value $<0.001)$, antibiotic use $(P$ value $<0.001)$ and admission ( $P$ value $<0.001)$ during the previous three months, parental smoking $(P$ value $<0.001)$ and sleeping with parents ( $P$ value 0.022 ) [24]. In this particular study, there was no host factor associated with MRSA carriage. This could be attributed to short period of time the study was conducted or to the small sample size.

\section{Conclusion}

There was high MRSA carriage, $39.1 \%$ prevalence and $50 \%$ incidence, among patients admitted in surgical ward. Resistance to commonly used antibiotics was high, $80 \%$ of the isolates were MDR. Therefore, there is the need for continuous surveillance to monitor aetiology and antimicrobial susceptibility pattern to guide the empirical use of antimicrobials, more studies exploring the role of colonization by MRSA in subsequent infections would also shed more light.

\section{Declarations}

\section{Ethics approval and consent to participate:}

Ethical approval to conduct the study was obtained from Mbarara University of Science and Technology Research and Ethics Committee (Ref. MUREC 1/6) to conduct this study. Upon ethical clearance, permission was sought from Mbarara Regional Referral Hospital to conduct research on admitted patients of a surgical ward and informed consent was sought from each participant. All participants who had MRSA were referred to clinicians for further management. 


\section{Consent for publication:}

Not applicable

\section{Conflict of interest:}

Authors declare that they have no conflicts of interest.

\section{Data Availability:}

Raw data of the study can by accessed by emailing mugimbapross@gmail.com

\section{Authors' contributions:}

$\mathrm{PM}$ and JB contributed in study conception, design and data Collection, $\mathrm{AB}$ and $\mathrm{HI}$ carried out data analysis. $\mathrm{PM}$ and $\mathrm{BM}$ wrote the first draft of the manuscript while $\mathrm{AB}$ reviewed the manuscript and JB supervised the whole research process.

\section{Funding:}

This was a private funding research and we did not receive any grant to fund this study.

\section{Acknowledgements:}

We appreciate the contribution and support from study participants and all Staff in the Department of microbiology Mbarara University of science and Technology.

\section{References}

1. Abbasi, S., et al., Colonization with Methicillin-Resistant Staphylococcus aureus uponIntensive Care Unit Admission: Incidence and Risk Factors.

2. Gitau, W., et al., Antimicrobial susceptibility pattern of Staphylococcus aureus isolates from clinical specimens at Kenyatta National Hospital. BMC research notes, 2018. 11(1): p. 226.

3. Alegre, M.-L., et al., Impact of Staphylococcus aureus USA300 colonization and skin infections on systemic immune responses in humans. The Journal of Immunology, 2016. 197(4): p. 1118-1126.

4. Uhlemann, A.-C., et al., Evolution of community-and healthcare-associated methicillin- resistant Staphylococcus aureus. Infection, genetics and evolution, 2014. 21: p. 563-574.

5. Jayatilleke, K. and P. Bandara, Antibiotic sensitivity pattern of Staphylococcus aureus in a tertiary care hospital of Sri Lanka. Sri Lankan Journal of Infectious Diseases, 2012. 2(2).

6. Abdulgader, S.M., et al., Molecular epidemiology of Methicillin-resistant Staphylococcus aureus in Africa: a systematic review. Frontiers in microbiology, 2015. 6: p. 348.

7. Egyir, B., et al., Molecular epidemiology and antimicrobial susceptibility of clinical Staphylococcus aureus from healthcare institutions in Ghana. PLoS One, 2014. 9(2): p. e89716. 
8. Stanley, I.J., et al., Prevalence and antibiotic susceptibility patterns of clinical isolates of Methicillinresistant staphylococcus aureus in a tertiary care Hospital in Western Uganda. British Microbiology Research Journal, 2014. 4(10): p. 1168-1177.

9. Aizen, E., et al., Risk factors for methicillin-resistant Staphylococcus aureus colonization in a geriatric rehabilitation hospital. The Journals of Gerontology Series A: Biological Sciences and Medical Sciences, 2007. 62(10): p. 1152-1156.

10. Kumar, N., et al., High S aureus colonization prevalence among patients with SSTIs and controls in an urban emergency department. Journal of Clinical Microbiology, 2014: p. JCM. 03221-14.

11. Sangeda, R.Z., et al., Antimicrobial susceptibility patterns of Staphylococcus aureus and coagulase negative staphylococci isolated from humans in Nairobi, Kenya. African Journal of Microbiology Research, 2017. 11(18): p. 705-711.

12. Bebell, L.M., et al., Prevalence and correlates of MRSA and MSSA nasal carriage at a Ugandan regional referral hospital. Journal of Antimicrobial Chemotherapy, 2016. 72(3): p. 888-892.

13. Ojulong, J., et al., Relative prevalence of methicilline resistant Staphylococcus aureus andlts susceptibility pattern in Mulago Hospital, Kampala, Uganda. Tanzania journal of health research, 2009. 11(3).

14. Schaumburg, F., et al., Transmission of Staphylococcus aureus between mothers and infants in an African setting. Clinical Microbiology and Infection, 2014. 20(6): p. 0390- 0396.

15. Witte, W., Community-acquired methicillin-resistant Staphylococcus aureus: what do weneed to know? Clinical Microbiology and Infection, 2009. 15: p. 17-25.

16. Butterly, A., U. Schmidt, and J. Wiener-Kronish, Methicillin-resistant Staphylococcus aureus colonization, its relationship to nosocomial infection, and efficacy of control methods.

Anesthesiology: The Journal of the American Society of Anesthesiologists, 2010. 113(6): p. 14531459.

17. Kahsay, A., et al., Isolation and antimicrobial susceptibility pattern of Staphylococcus aureus in patients with surgical site infection at Debre Markos Referral Hospital, Amhara Region, Ethiopia. Archives of public Health, 2014. 72(1): p. 16.

18. Aminaka, M., et al., Antimicrobial Susceptibility of Clinical Isolated Methicillin-Resistant Staphylococcus aureus (MRSA) in Japanese Hospitals. American Journal of Infection Control, 2007. 35(5): p. E14.

19. Mermel, L.A., et al., Methicillin-resistant Staphylococcus aureus colonization at different body sites: a prospective, quantitative analysis. Journal of Clinical Microbiology, 2011. 49(3): p. 1119-1121.

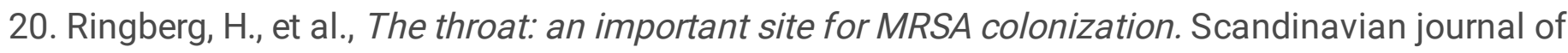
infectious diseases, 2006. 38(10): p. 888-893.

21. Sassmannshausen, R., et al., MRSA prevalence and associated risk factors among health-care workers in non-outbreak situations in the Dutch-German EUREGIO. Frontiers in microbiology, 2016. 7: p. 1273. 
22. Bradley, F., Eradication or decolonization of methicillin-resistant Staphylococcus aureus carriage: what are we doing and why are we doing it?, 2007, The University of Chicago Press.

23. Seni, J., et al., Molecular characterization of Staphylococcus aureus from patients with surgical site infections at Mulago Hospital in Kampala, Uganda. PLoS One, 2013. 8(6): p. e66153.

24. Soltani, B., et al., Risk factors for methicillin resistant Staphylococcus aureus nasalcolonization of healthy children. Jundishapur journal of microbiology, 2014. 7(9).

\section{Figures}

\section{Prevalence of MRSA ( $\mathrm{N}=46)$}

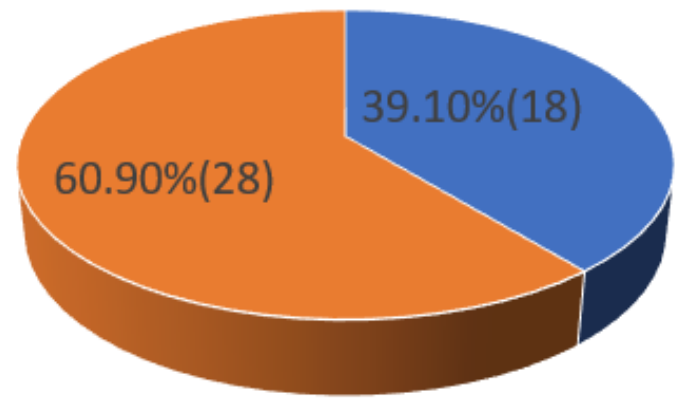

- MRSA - Negative

\section{Figure 1}

showing prevalence of MRSA carriage prior to admission. 


\section{Incidence of MRSA(N=26)}

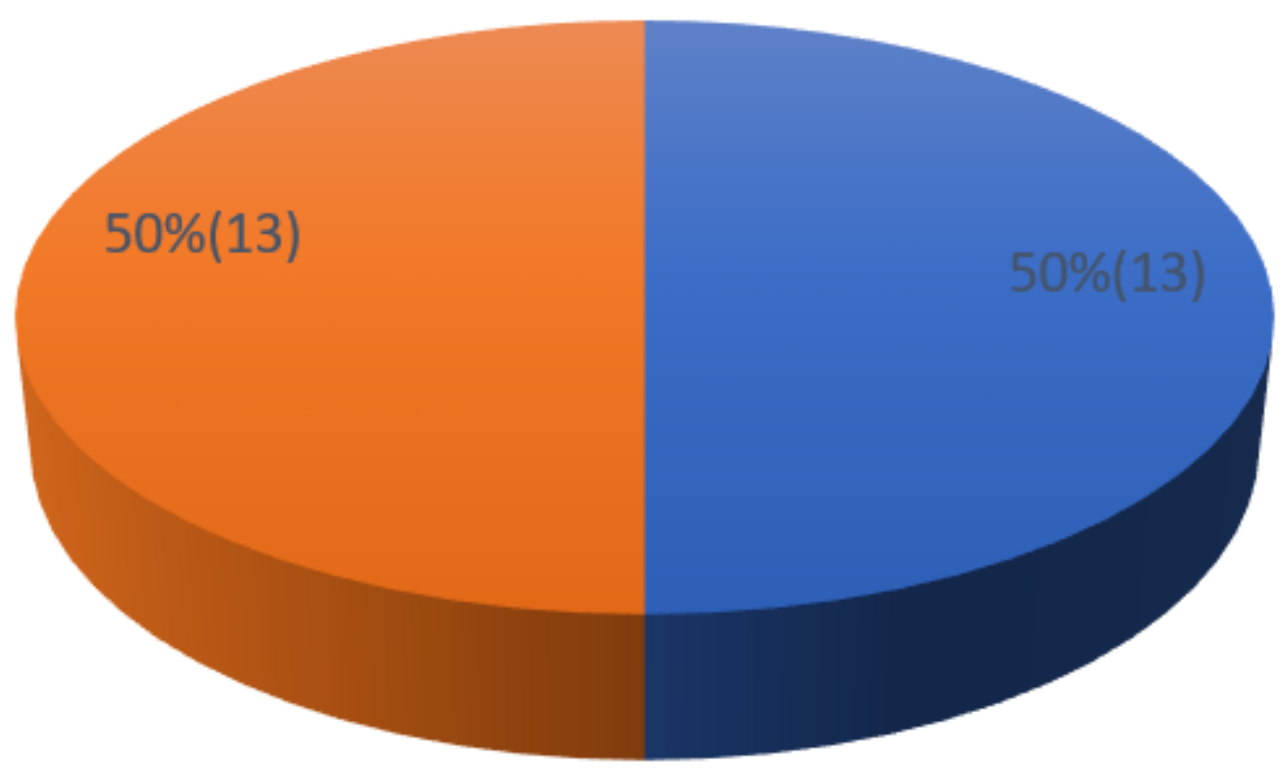

MRSA positive Negative

Figure 2

showing incidence of MRSA carriage during hospitalisation 


\section{DST patterns for isolated S. aureus.}

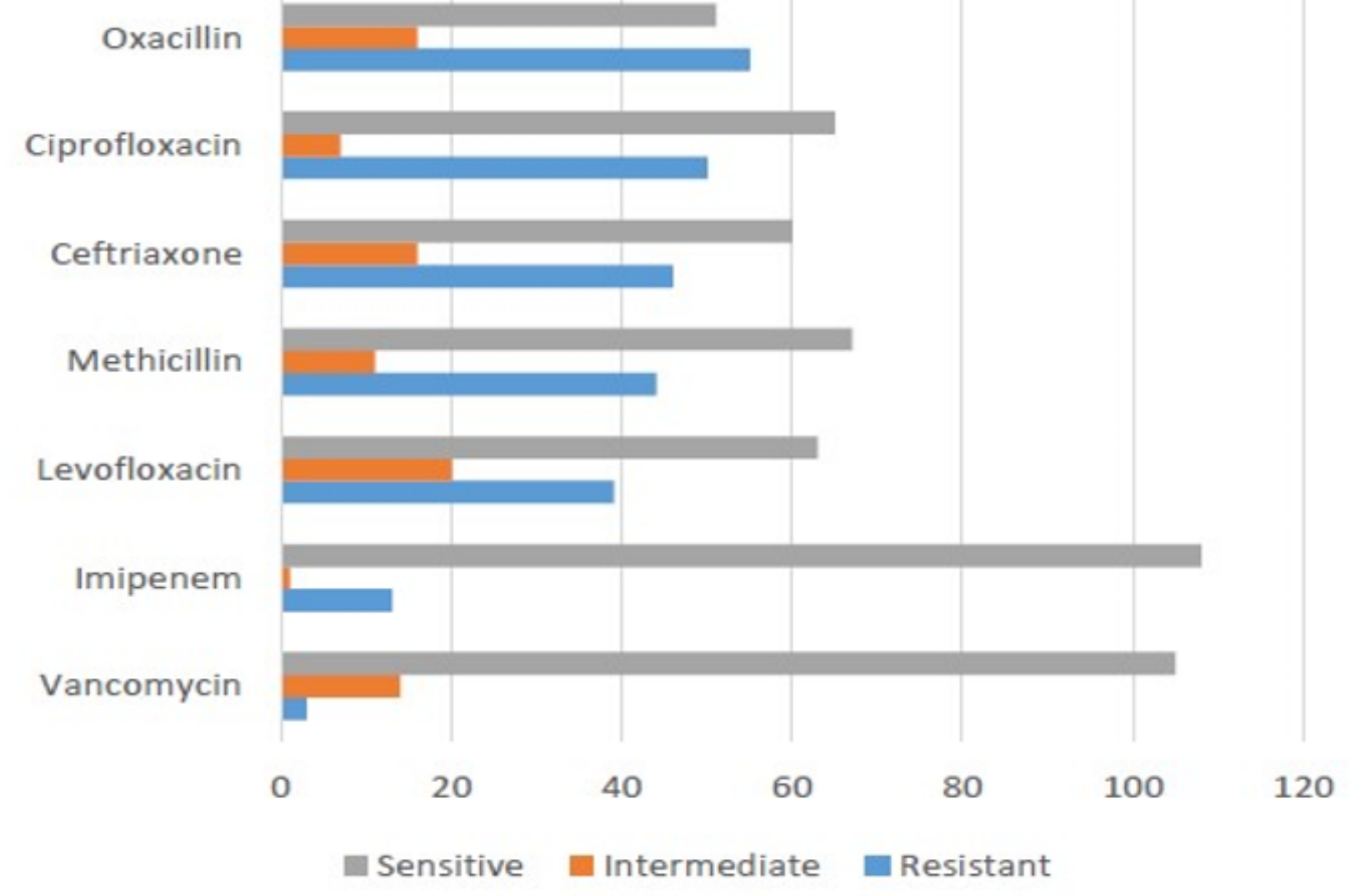

Figure 3

DST patterns for $\mathrm{S}$. aureus isolate 
MRSA colonization by site.

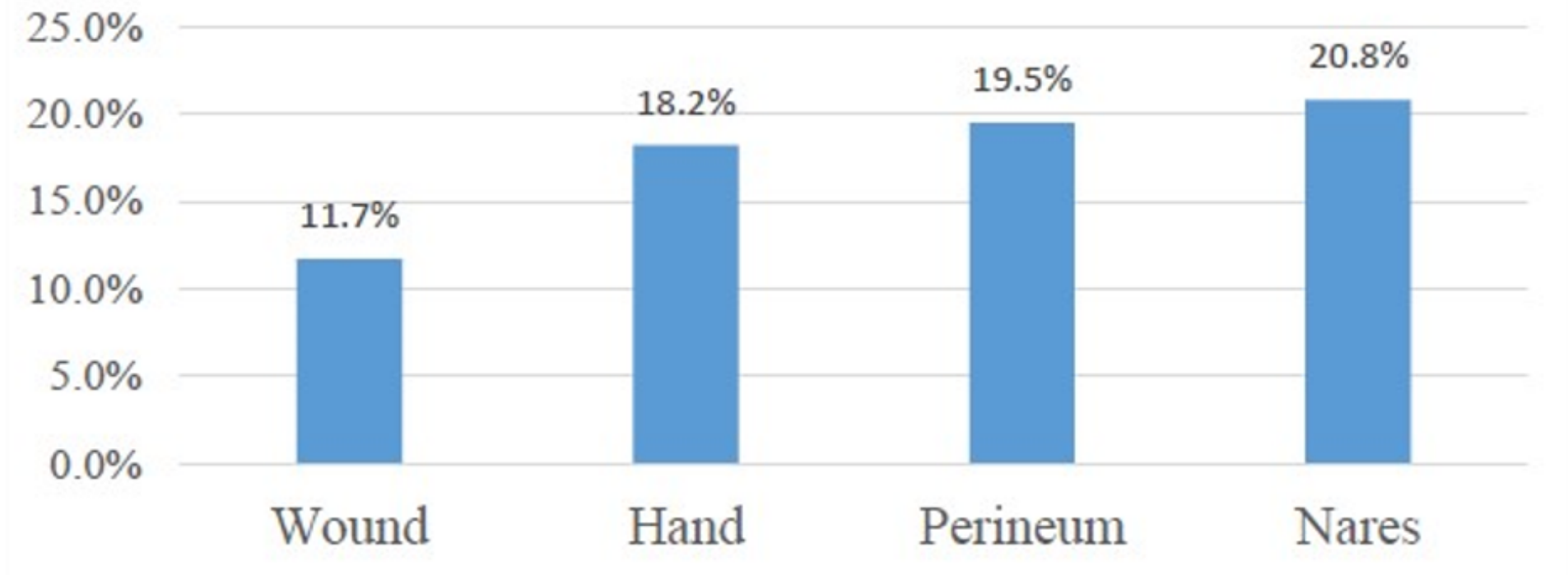

Figure 4

showing MRSA Carriage by site 